ARTIGO ORIGINAL

\title{
Nitrato e sulfato na precipitação pluviométrica em povoamento de Eucalyptus saligna Smith. no bioma Pampa
} \author{
biome \\ Grasiele Dick ${ }^{1}$ (D), Mauro Valdir Schumacher ${ }^{1}$ (D), Elias Frank de Araújo² (D) \\ ${ }^{1}$ Universidade Federal de Santa Maria - UFSM, Santa Maria, RS, Brasil \\ ${ }^{2}$ Empresa CMPC, Guaíba, RS, Brasil
}

Nitrate and sulfate in rainfall in Eucalyptus saligna Smith. stand in the Pampa

Como citar: Dick, G., Schumacher, M. V., \& Araújo, E. F. (2020). Nitrato e sulfato na precipitação pluviométrica em povoamento de Eucalyptus saligna Smith. no bioma pampa. Scientia Forestalis, 48(127), e3232. https://doi.org/10.18671/scifor.v48n127.02

\begin{abstract}
Resumo
A precipitação pluviométrica que incide sobre o dossel e escoa pelos troncos das árvores de eucalipto é uma importante via da ciclagem geoquímica, que contribui à nutrição florestal. O objetivo deste estudo é caracterizar o aporte de nitrato (N) e sulfato/enxofre (S) pela precipitação pluviométrica e a hipótese testada é a de que a precipitação interna (Pi) e o escoamento pelo tronco (Et) aumentam a concentração desses elementos quando comparada à precipitação incidente $(P)$ em povoamento de Eucalyptus saligna Smith., cultivado na região central do Rio Grande do Sul, bioma Pampa. Durante o ano de 2012, foram quantificados os volumes e as concentrações de nitrato e sulfato contidas na solução da precipitação pluviométrica $(\mathrm{P})$, precipitação interna $(\mathrm{Pi})$ e escoamento pelo tronco $(\mathrm{Et})$. O volume total de precipitação foi de $1.224,7 \mathrm{~mm}$, sendo que $15,9 \%$ foi interceptado pelas copas. A concentração de $\mathrm{N}$ é maior na $\mathrm{Pi}$, seguida de $\mathrm{P}$ e Et, ao passo que a forma de distribuição da precipitação não influencia a concentração de S. Há relevante contribuição da Pi para o aporte de $\mathrm{N}$ e de $\mathrm{P}$ para entrada de $\mathrm{S}$, de forma que as recomendações futuras de aplicação de fertilizantes podem ser otimizadas por meio da redução de até $36,8 \%$ de N, evitando-se assim dispêndios de nutrientes e redução de custos.
\end{abstract}

Palavras-chave: Ciclagem dos nutrientes; Nutrição florestal; Hidrologia florestal.

\begin{abstract}
The rainfall that drops on the canopy and flows along the trunks of eucalyptus trees is an important path of geochemical cycling that contributes to forest nutrition. The objective of this study is to characterize the contribution of nitrate $(\mathrm{N})$ and sulfate / sulfur $(\mathrm{S})$ by the rainfall and the hypothesis tested is that the through fall $(\mathrm{Pi})$ and stemflow increase the concentration of these elements when compared to the incident rainfall $(P)$ on a Eucalyptus saligna Smith. stand, growing in the central region of Rio Grande do Sul state, Pampa biome. During 2012, the volumes and concentrations of nitrate and sulfate contained in the incident rainfall solution $(\mathrm{P})$ throughfall $(\mathrm{Pi})$ and stemflow $(\mathrm{Et})$ were quantified. The total volume of rainfall was $1,224.7 \mathrm{~mm}$, where $15.9 \%$ was intercepted by the canopies. The concentration of $\mathrm{N}$ is greater in $\mathrm{Pi}$, followed by $\mathrm{P}$ and $\mathrm{Et}$, whereas the distribution of them does not influence the sulfate concentration. There was a relevant contribution of $\mathrm{Pi}$ to the $\mathrm{N}$ and $\mathrm{P}$ on the $\mathrm{S}$ input; so that any future recommendation about fertilization can be optimized by reduction of $36.8 \%$ in $\mathrm{N}$; therefore avoiding a nutrient excess and by reducing costs.
\end{abstract}

Keywords: Nutrient cycling; Forest nutrition; Forest hydrology.

Fonte de financiamento: Nenhuma.

Conflito de interesse: Nada a declarar.

Autor correspondente: grasidick@hotmail.com

Recebido: 21 dezembro 2018.

Aceito: 30 junho 2019

Editor: Francides Gomes Silva Júnior.

(c) Este é um artigo publicado em acesso aberto (Open Access) sob a licença Creative Commons Attribution, que permite uso, distribuição e reprodução ey em qualquer meio, sem restrições desde que o trabalho original seja corretamente citado. 


\section{INTRODUÇÃO}

A ciclagem de nutrientes é um processo importante para a manutenção da produtividade em povoamentos de eucalipto, podendo prover o suprimento nutricional ao desenvolvimento da vegetação (Poggiani \& Schumacher, 2005). Este processo ecológico é importante para a contínua circulação de nutrientes no sítio florestal, ao passo que, o entendimento desses fluxos possibilita a definição de manejos operacionais e silviculturais eficientes (Schumacher \& Viera, 2015).

A ciclagem geoquímica é uma importante via de incorporação de nutrientes em povoamentos florestais. É caracterizada pela entrada via deposição atmosférica úmida e seca, oriunda do depósito de partículas/nutrientes sobre o dossel que é carreado através da precipitação pluviométrica incidente, que lava as copas e troncos das árvores (Chang, 2006). Caracterizar essas entradas de minerais em povoamentos florestais, via precipitação interna e escoamento pelo tronco, é primordial à silvicultura e, por meio do monitoramento da ciclagem geoquímica, pode-se otimizar a fertilização florestal, reduzir custos e operações, aliando a produção florestal e conservação de recursos naturais (Schumacher \& Viera, 2015).

A água da precipitação pluviométrica não é pura, pois contêm substâncias químicas derivadas de fontes de gases-traço, como óxidos de enxofre e nitrato, aerossóis e partículas de poeiras de incêndios, vulcões e vendavais, que são ricas em sódio, magnésio, cloreto e sulfato de cálcio, potássio, sulfato, dentre outros elementos (Begon et al., 2007). O acúmulo desse particulado, poeira e cinzas sobre o dossel é carreado após a interceptação da água pelo folhedo, podendo influenciar a concentração de íons na solução aquosa da precipitação pluviométrica interna e do escoamento pelo tronco nos povoamentos florestais (Shen et al., 2013; Ashagrie \& Zech, 2010; Laclau et al., 2010).

A hipótese testada neste estudo é a de que a precipitação interna e o escoamento pelo tronco aumentam a concentração de nitrato e sulfato quando comparadas à precipitação incidente em povoamento florestal. O objetivo deste estudo é caracterizar o aporte de nitrato $(\mathrm{N})$ e sulfato/enxofre $(\mathrm{S})$ pela precipitação pluviométrica e avaliar se as diferentes formas de distribuição da precipitação pluviométrica (incidente, interna e escoamento pelo tronco) influenciam a concentração desses elementos, em povoamento de Eucalyptus saligna cultivado na região central do Rio Grande do Sul, bioma Pampa.

\section{MATERIAL E MÉTODOS}

\section{Área de estudo}

O estudo foi realizado em um povoamento de Eucalyptus saligna Smith. cultivado no município de São Gabriel, região central do estado do Rio Grande do Sul, Brasil (coordenadas geográficas centrais $30^{\circ} 30^{\prime} 12^{\prime \prime} \mathrm{S}$ e $\left.54^{\circ} 10^{\prime} 0,8^{\prime \prime} \mathrm{O}\right)$. O relevo da área é suavemente ondulado, em altitude de $114 \mathrm{~m}$ em relação ao nível médio do mar, onde o solo é do tipo Cambissolo Háplico Distrófico típico, de média textura, pH ácido, baixos teores de matéria orgânica e nutrientes (Salvador et al., 2014).

O clima da região, segundo a classificação de Köppen, é do tipo Cfa subtropical úmido, com verão quente e sem estação seca. A temperatura média do mês mais quente é superior a $22^{\circ} \mathrm{C}$ e, no mês mais frio as temperaturas variam entre $-3^{\circ} \mathrm{C}$ a $18^{\circ} \mathrm{C}$. Quanto à precipitação pluviométrica, a mínima no mês mais seco é superior a $40 \mathrm{~mm}$ e a precipitação média anual pode variar de 1.600 a $1.900 \mathrm{~mm}$ (Alvares et al., 2013). No diagrama climático do município de São Gabriel (Figura 1), elaborado a partir de dados médios de temperatura e precipitação (1960-1990) (Instituto Nacional de Meteorologia, 2016), pode-se observar que na região as temperaturas mais baixas ocorrem nos meses de junho, julho e agosto (estação do inverno) e as mais altas no período do verão, de novembro a fevereiro. A precipitação é bem distribuída ao longo do ano, no entanto, com menor incidência na estação mais quente. 


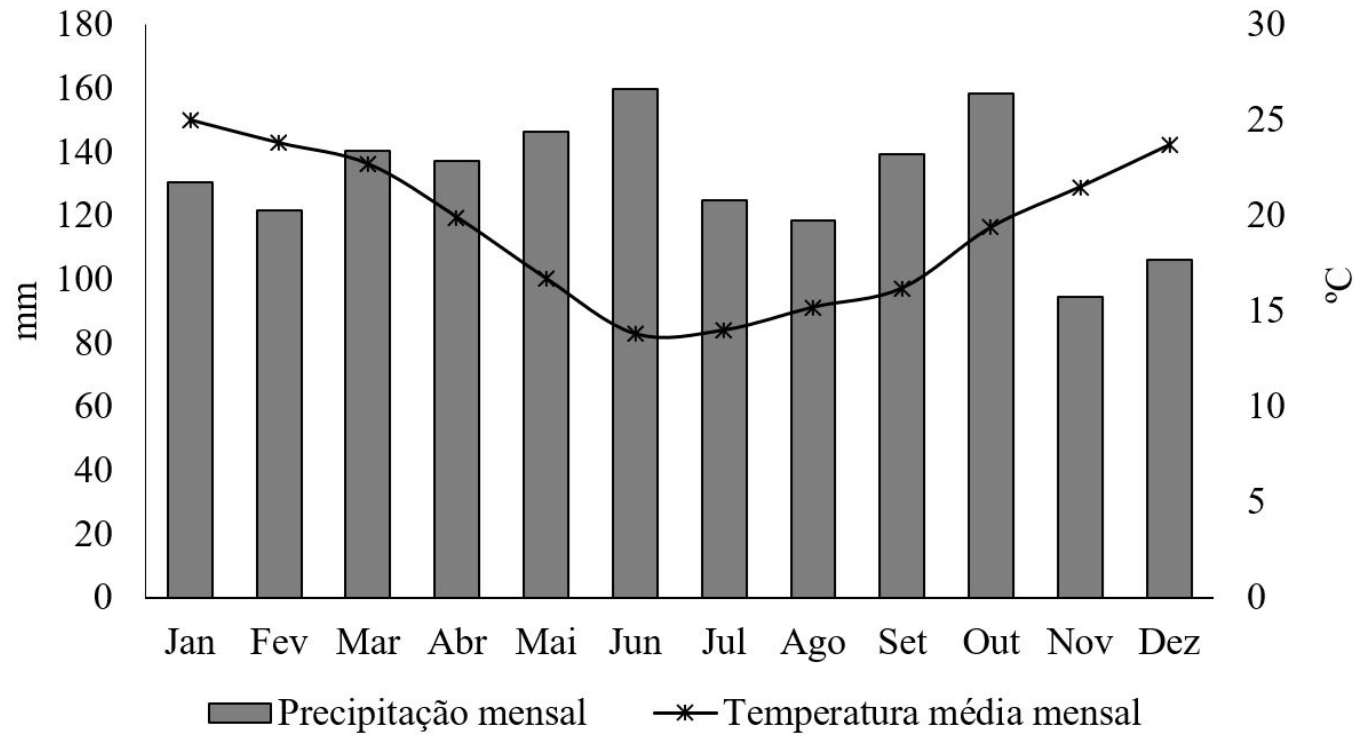

Figura 1 - Diagrama climático do município de São Gabriel, Rio Grande do Sul (1960-1990). Fonte: Adaptado de Instituto Nacional de Meteorologia (2016)

A implantação do povoamento de Eucalyptus saligna ocorreu em 2007, com o plantio das mudas clonais no espaçamento $3,5 \mathrm{~m} \times 2,75 \mathrm{~m}$, onde o solo foi preparado com subsolagem até $50 \mathrm{~cm}$ de profundidade, calagem (2,0 $\mathrm{Mg} \mathrm{ha}^{-1}$ de calcário dolomítico) e adubação fosfatada no sulco (500 kg ha-1 fosfato reativo). A adubação de plantio foi realizada com $100 \mathrm{~g} \mathrm{planta}^{-1}$ de N- $\mathrm{P}_{2} \mathrm{O}_{5}-\mathrm{K}_{2} \mathrm{O}$ (06:30:06), com posterior cobertura aos 120 dias com 200 g planta-1 de N- $\mathrm{P}_{2} \mathrm{O}_{5}-$ $\mathrm{K}_{2} \mathrm{O}(12: 00: 12)+0,5 \% \mathrm{~B}$ e aos 365 dias com 200 g planta-1 de N- $\mathrm{P}_{2} \mathrm{O}_{5}-\mathrm{K}_{2} \mathrm{O}$ (12:00:20). Em média, são aplicados $70 \mathrm{~kg} \mathrm{ha}^{-1}$ de nitrogênio no primeiro ano da implantação e não há adição de enxofre na fertilização mineral.

\section{Amostragem da precipitação pluviométrica}

Durante o ano de 2012 (janeiro a dezembro), cinco anos após o plantio, realizou-se o monitoramento do volume e das concentrações de nitrato e sulfato contidas na precipitação incidente que atinge as copas das árvores $(\mathrm{P})$, na precipitação interna $(\mathrm{Pi})$ e no escoamento pelo tronco (Et) do povoamento de Eucalyptus saligna (Figura 2a).
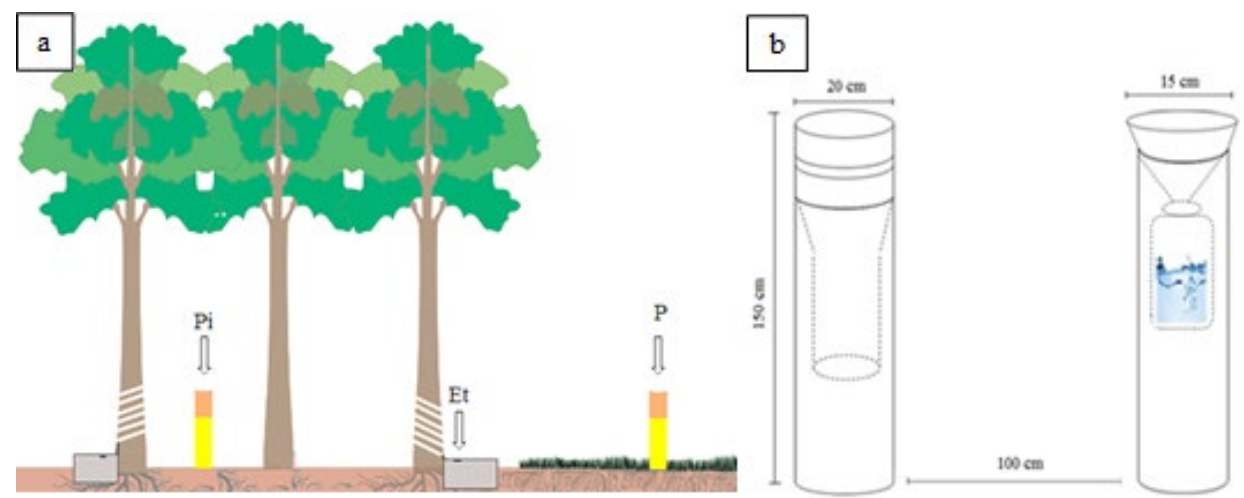

Figura 2 - a) Distribuição dos coletores de precipitação incidente (P), precipitação interna (Pi) e escoamento pelo tronco (Et); b) Detalhe dos coletores. Fonte: Adaptado de Dick et al. (2018).

Para a quantificação do volume de precipitação (P e Pi), conforme metodologia descrita em Dick et al. (2018), foram utilizados coletores de PVC com diâmetro de captação de $20 \mathrm{~cm}$ e, para a análise química, utilizaram-se coletores constituídos por recipiente plástico com 
capacidade de armazenamento de $1 \mathrm{~L}$, totalmente vedados e fixados em um funil, com diâmetro de $15 \mathrm{~cm}$ (Figura 2b). Os coletores, apoiados em tubos de PVC, foram distanciados a $1,50 \mathrm{~m}$ do nível do solo e equidistantes em 1,0 m. O escoamento pelo tronco (Et) foi monitorado por meio de coletores constituídos por mangueira (fixada na árvore com espuma de poliuretano e cola de silicone) e recipiente coletor, com capacidade para estoque de 60L, instalados ao redor do tronco de árvores que possuíam diâmetro à altura do peito médio do povoamento $(20 \mathrm{~cm})$, à altura de $1,50 \mathrm{~m}$ do nível do solo.

A análise da $\mathrm{P}$ foi realizada por meio da instalação de três coletores para quantificação de volume $(n=3)$ e três para análise da concentração $(n=3)$, localizados em área aberta, a $50 \mathrm{~m}$ de distância do povoamento. Para o monitoramento da Pi foram instaladas quatro parcelas $\left(420 \mathrm{~m}^{2}\right)$, distribuídas de forma aleatória no interior do povoamento, onde em cada uma delas foram instalados três coletores para análise de volume $(n=12)$ e três para concentração $(n=12)$, dispostos na linha, entrelinha e diagonal entre árvores, segundo descrição em Dick et al. (2018). O volume de precipitação direcionado ao escoamento pelo tronco (Et) foi mensurado por meio de três coletores, também instalados em cada uma dessas quatro parcelas $(n=12)$, de onde se retiraram as alíquotas para análise química.

Para minimizar a influência da temperatura, vento, deposição de partículas e insetos, dentre outras fontes de variação, nos coletores de $\mathrm{P}$ e $\mathrm{Pi}$ destinados à coleta das amostras para análises químicas, foram colocados filtros porosos $(0,45 \mu \mathrm{m})$ no funil acoplado ao recipiente coletor, de modo a permitir apenas a passagem da solução aquosa. O coletor foi totalmente vedado e circundado por cano de PVC, para evitar perdas por evaporação, formação de algas e contaminações. Em todas as coletas se manteve o cuidado com a assepsia dos coletores, que eram lavados com água deionizada depois de retirada a amostra e, nos coletores de $\mathrm{P}$, que ficaram em área aberta, foram fixadas estruturas com pontas de aço galvanizado, evitando pousio de aves e contaminação de amostras.

Quinzenalmente quantificou-se o volume de precipitação e coletou-se uma alíquota de cada coletor destinado à análise química da solução aquosa da P, Pi e Et. As amostras quinzenais eram acondicionadas em potes esterilizados de plástico com tampa, transportados em caixa térmica e acondicionados em ambiente refrigerado (com temperatura variando de 0 a $5^{\circ} \mathrm{C}$ ) até o processamento mensal, com posterior junção das duas amostras quinzenais de cada coletor para formação de amostra composta. Em laboratório, as amostras compostas mensais de solução aquosa da $\mathrm{P}$, Pi e Et passaram por filtro de poros de $0,45 \mu \mathrm{m}$, com posterior determinação das concentrações de nitrato $\left(\mathrm{N}^{-\mathrm{NO}_{3}}{ }^{-}\right)$e sulfato $\left(\mathrm{SO}_{4}{ }^{2-}\right)$, por meio de cromatografia iônica (Metrohm 861 Advanced Compac IC), segundo a metodologia proposta pela American Public Health Association (1998).

Para cálculo do volume de precipitação pluviométrica $(\mathrm{mm})$ em cada forma de distribuição da precipitação, foi adotada a expressão: $P t=V / a c$, em que: $\mathrm{P}=$ precipitação pluviométrica $(\mathrm{mm}), \mathrm{V}=$ volume coletado $(\mathrm{L}), \mathrm{ac}=$ área do coletor $\left(\mathrm{m}^{2}\right)(\mathrm{Sch}$ umacher \& Viera, 2015). Para determinação da quantidade de enxofre aportado (S), multiplicou-se a quantidade de sulfato $\left(\mathrm{S}_{-} \mathrm{SO}_{4}{ }^{2-}\right.$ ) pela constante 0,3338 . Para a determinação das quantidades ( $\mathrm{kg} \mathrm{ha}^{-1} \mathrm{ano}^{-1}$ ) de nitrato e enxofre aportados, calculou-se o produto entre a concentração $\left(\mathrm{mg} \mathrm{L}^{-1}\right)$ do elemento e o volume de precipitação para cada mês, com posterior soma anual.

\section{Análise dos dados}

Os dados individuais de concentração de nitrato e sulfato passaram por transformação logarítmica, visando à distribuição normal e a homogeneidade de variâncias, que foram verificadas pelos testes de Shapiro-Wilk e Bartlett, respectivamente.

Os dados foram submetidos à análise de variância (ANOVA) pelo teste $F$, no delineamento inteiramente casualizado. Havendo significância do teste $F$, as médias foram comparadas pelo teste Scott-Knott $(a=5 \%)$, com diferentes números de repetições, para detecção de diferenças entre as concentrações médias mensais de cada elemento (nitrato e sulfato) em função da forma de distribuição da precipitação (P, Pi e Et) no povoamento florestal. 


\section{RESULTADOS E DISCUSSÃO}

\section{Distribuição da precipitação}

Durante o ano de 2012 o volume total da precipitação pluviométrica incidente na plantação de Eucalyptus saligna foi de $1.224,7 \mathrm{~mm}$, com máxima em outubro e mínima em maio. As copas das árvores interceptaram 15,3\% deste volume, ao passo que somente 3,0\% da precipitação escoou pelo tronco (Figura 3).

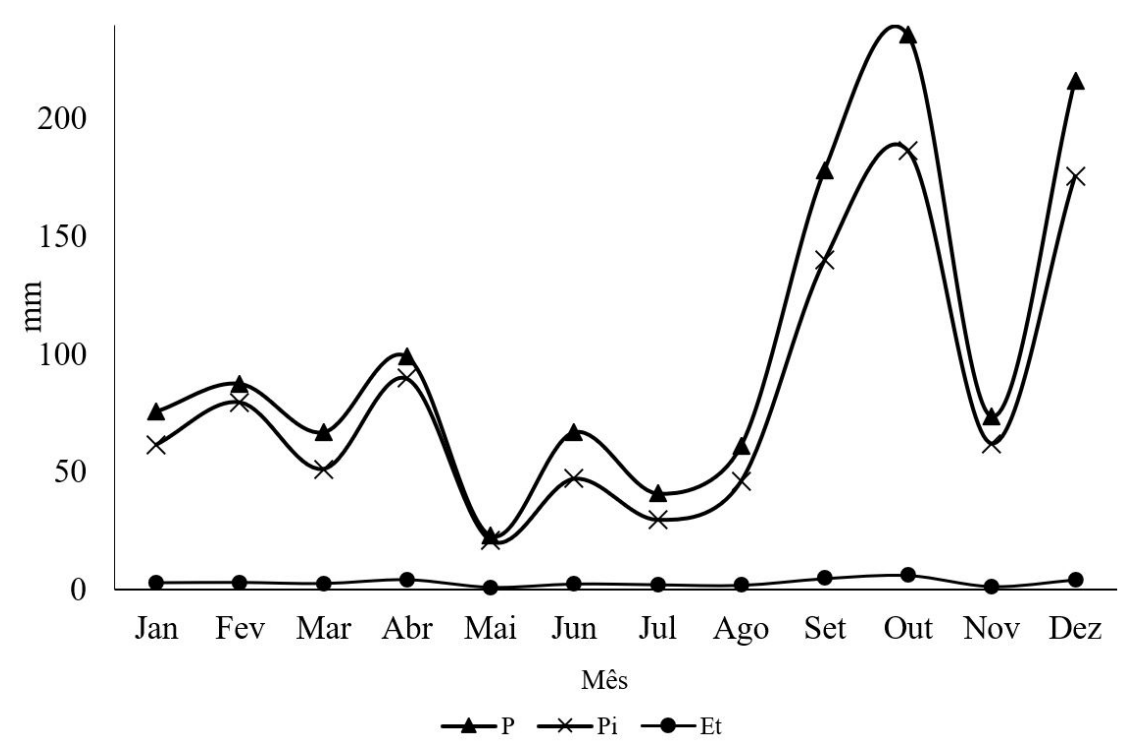

Figura 3 - Distribuição da precipitação pluviométrica em povoamento de Eucalyptus saligna na região central do Rio Grande do Sul, bioma Pampa. $P$ = precipitação incidente $(n=3)$; $\mathrm{Pi}=$ precipitação interna $(n=12) ;$ Et = escoamento pelo tronco $(n=12)$.

A precipitação pluviométrica foi bem distribuída ao longo do ano, no entanto, o volume incidente está abaixo dos valores registrados para a região (Figura 1). Isso ocorreu, pois durante o período do estudo houve influência do fenômeno "La Niña", que atuou no Brasil no período de dezembro de 2011 a março de 2012, ocasionando chuva abaixo da média para o estado do Rio Grande do Sul (Melo, 2011).

Neste estudo, onde as árvores de Eucalyptus saligna estão com cinco anos e o dossel está fechado, a interceptação de $15,3 \%$ da precipitação pelas copas da plantação é um valor considerado dentro do esperado para o tipo de vegetação, semelhante ao observado em Eucalyptus grandis aos três anos (Sari et al., 2016). No entanto, à medida que há o crescimento da árvore, o índice de área foliar e a proporção de biomassa alocada nas folhas reduzem, consequentemente, a interceptação da precipitação pluviométrica é menor (Salvador et al., 2016; Almeida et al., 2007; Lima, 1976).

Além do fator idade e índice de área foliar, a quantidade de chuva interceptada também varia em função da espécie, arquitetura de copa e espaçamento de plantio (Diniz et al., 2013; Chang, 2006; Almeida et al., 2007). Por exemplo, em povoamentos de pinus a arquitetura de copa piramidal, maior índice de área foliar e disposição dos galhos aumentam a interceptação da precipitação pluviométrica pelo dossel (Lima, 1976), que é expressivamente maior quando comparada às florestas nativas e às plantações de Eucalyptus (Giglio \& Kobiyama, 2013).

\section{Concentrações de nitrato e sulfato na precipitação pluviométrica}

Em todas as formas de distribuição da precipitação pluviométrica há maior concentração de sulfato do que nitrato. A forma de distribuição da precipitação influenciou somente a concentração de nitrato, que é maior na precipitação interna e não há efeito da lavagem das copas (Pi) e escoamento pelo tronco sobre a concentração de sulfato (Tabela 1). 
Tabela 1 - Concentrações de nitrato $\left(\mathrm{N}-\mathrm{NO}_{3}{ }^{-}\right)$e sulfato $\left(\mathrm{SO}_{4}{ }^{2-}\right)$ na precipitação pluviométrica incidente $(\mathrm{P})$, precipitação interna $(\mathrm{Pi})$ e escoamento pelo tronco $(\mathrm{Et})$ em povoamento de Eucalyptus saligna na região central do Rio Grande do Sul, bioma Pampa.

\begin{tabular}{|c|c|c|c|c|c|c|}
\hline \multirow{3}{*}{ Mês } & \multicolumn{6}{|c|}{$\mathrm{mg} \mathrm{L}^{-1}$} \\
\hline & \multicolumn{3}{|c|}{$\mathrm{N}-\mathrm{NO}_{3}^{-}$} & \multicolumn{3}{|c|}{$\mathrm{SO}_{4}{ }^{2-}$} \\
\hline & $\mathbf{P}$ & $\mathbf{P i}$ & Et & $\mathbf{P}$ & $\mathbf{P i}$ & Et \\
\hline Jan & $0,23 b^{*}$ & $0,58 a$ & $0,18 b$ & $0,71 a$ & $0,82 a$ & $0,66 a$ \\
\hline Fev & $0,26 b$ & $0,72 a$ & $0,26 b$ & $0,39 b$ & $0,61 a$ & $0,42 b$ \\
\hline Mar & $0,20 a$ & $0,30 a$ & $0,00 \mathrm{~b}$ & $1,40 \mathrm{~b}$ & $1,93 b$ & $2,93 a$ \\
\hline Abr & $0,12 b$ & $0,40 a$ & $0,18 b$ & $0,55 b$ & $1,43 a$ & $0,43 b$ \\
\hline Mai & $0,34 b$ & $1,14 a$ & $1,09 a$ & $2,46 a$ & $1,60 \mathrm{~b}$ & $2,17 a$ \\
\hline Jun & $0,20 c$ & $0,78 a$ & $0,40 b$ & $1,12 a$ & $1,31 a$ & $0,79 b$ \\
\hline Jul & $0,10 a$ & $0,07 a$ & $0,05 a$ & $1,07 a$ & $1,08 a$ & $1,05 a$ \\
\hline Ago & $0,25 b$ & $0,77 a$ & $0,62 a$ & $1,15 a$ & $1,16 a$ & $0,97 a$ \\
\hline Set & $0,50 b$ & $0,71 a$ & $0,43 b$ & $1,77 a$ & $1,77 a$ & $1,73 a$ \\
\hline Out & $0,11 b$ & $0,24 a$ & $0,03 b$ & $0,64 a$ & $0,64 a$ & $0,55 a$ \\
\hline Nov & $0,31 b$ & $0,93 a$ & $0,22 b$ & $0,83 a$ & $1,18 a$ & $1,19 a$ \\
\hline Dez & $0,02 a$ & $0,00 b$ & $0,00 b$ & $0,44 a$ & $0,44 a$ & $0,34 a$ \\
\hline Média mensal & $0,22 b$ & $0,55 a$ & $0,29 b$ & $1,04 a$ & $1,16 a$ & $1,10 a$ \\
\hline
\end{tabular}

*Médias iguais para as concentrações de cada elemento, não diferem entre as vias de entrada P $(n=3)$, Pi $(n=12)$ e Et $(n=12)$, pelo teste Scott-Knott, ao nível de 5\% de probabilidade de erro.

O aumento em $78,6 \%$ na concentração de nitrato na água da precipitação interna é resultado da interação da precipitação incidente com o dossel, que lixivia os elementos constituintes dos tecidos vegetais e a deposição seca das partículas de poeira e fuligem sobre as folhas e galhos, somada à deposição úmida (Shen et al., 2013). Essas partículas depositadas sobre o dossel são oriundas de queimadas da vegetação, que libera grande quantidade de particulados à atmosfera (Laclau et al., 2003) e deriva de fertilizantes aplicados nas lavouras e pastagens, perenes e anuais, que são cultivadas no Pampa e circundam os povoamentos florestais no bioma (Dick et al., 2018).

O aumento na concentração de íons na precipitação incidente após o contato com as copas das árvores ocorre em povoamentos de Eucalyptus dunnii (Dick et al., 2018), Eucalyptus grandis (Laclau et al., 2010), Eucalyptus sp. (Laclau et al., 2003), Eucalyptus globulus (Ashagrie \& Zech, 2010), dentre outros. No entanto, em alguns casos, a concentração pode reduzir após a interação da água com o dossel, pois alguns elementos contidos na precipitação pluviométrica são diretamente absorvidos pelas folhas (Carnol \& Bazgir, 2013).

No presente estudo, no qual a precipitação registrada está abaixo da média (Figura 3), as concentrações de nitrato e sulfato, em todas as formas de distribuição são maiores do que aquelas observadas em plantação de Eucalyptus dunnii, na região oeste do bioma Pampa, no entanto, estas foram avaliadas quando houve maior volume anual de precipitação pluviométrica (Dick et al., 2018).

As concentrações de íons na solução aquosa variaram de acordo com o volume da precipitação pluviométrica, assim, a concentração de sulfato e nitrato foi inversamente proporcional à quantidade de água em alguns meses de observação. Por exemplo, as concentrações de sulfato e nitrato na solução aquosa são menores nos meses de outubro e dezembro, quando houve maior volume de precipitação pluviométrica; já em maio e julho, quando o volume de precipitação ficou abaixo de $50 \mathrm{~mm}$, registraram-se as maiores concentrações de sulfato na P e Et e de $\mathrm{N}$ na Pi e Et (Tabela 1). Estes resultados evidenciam que quanto menor é o volume de precipitação, menor é a diluição dos nutrientes em água e, consequentemente, maiores serão as concentrações (Chang, 2006).

Altas concentrações de nitrato e sulfato na precipitação pluviométrica são indicativas de poluição atmosférica (Wu et al., 2013). Na região central do Rio Grande do Sul, no bioma Pampa, a queima da vegetação para renovação das pastagens é uma prática comum que lança particulados à atmosfera e, estudos já relataram que as altas concentrações de sulfato 
na precipitação incidente em Eucalyptus sp. estão associadas à prática da queima intensa de biomassa (Laclau et al., 2003).

\section{Aporte de nitrato e enxofre via precipitação pluviométrica}

As maiores quantidades ( $\mathrm{kg} \mathrm{ha}^{-1} \mathrm{ano}^{-1}$ ) de nitrato foram aportadas pela $\mathrm{Pi}$, seguida de $\mathrm{Pe}$ Et. Pela P houve maior aporte de enxofre, seguida pela Pi e Et. O Et contribui com menor quantidade de nutrientes à ciclagem geoquímica em função do menor volume de água que é direcionada ao tronco (Figura 4).

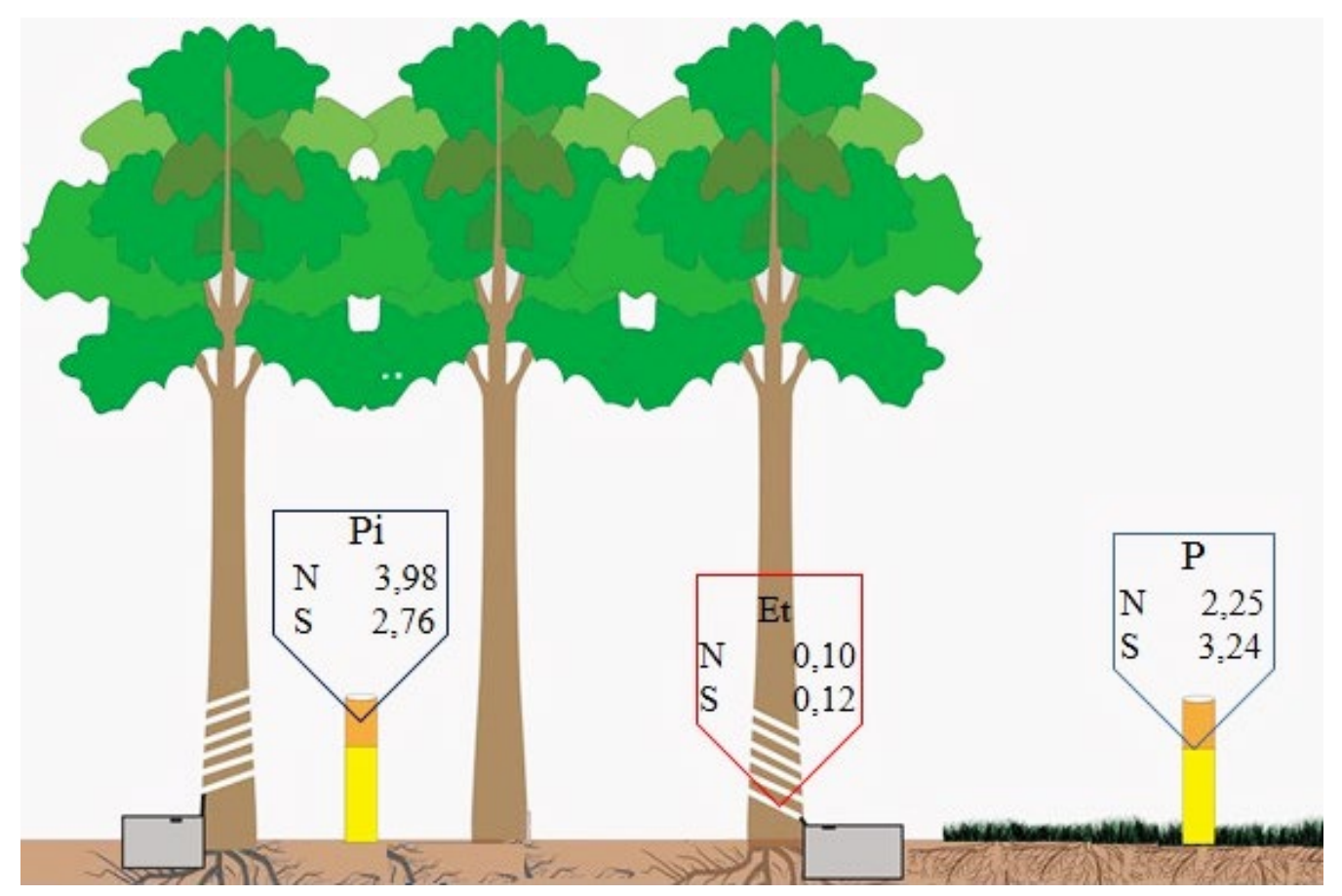

Figura 4 - Aporte $\left(\mathrm{kg} \mathrm{ha}^{-1} \mathrm{ano}^{-1}\right)$ de nitrato $(\mathrm{N})$ e enxofre $(\mathrm{S})$ pela precipitação pluviométrica incidente $(\mathrm{P})$, precipitação interna $(\mathrm{Pi})$ e escoamento pelo tronco (Et) em povoamento de Eucalyptus saligna na região central do Rio Grande do Sul, bioma Pampa.

Por meio da lavagem das copas das árvores ocorreu enriquecimento de $76,8 \%$ na quantidade de nitrato aportada no interior do povoamento (Pi). Considerando a entrada anual de 3,98 kg ha-1 de $\mathrm{N}$, para um ciclo de corte das árvores após sete anos de cultivo, pela Pi haverá aporte de mais de $27 \mathrm{~kg} \mathrm{ha}^{-1}$ de nitrato, o que corresponde a 38,6\% da quantidade do fertilizante mineral aplicada no plantio das mudas de Eucalyptus saligna. Visando mitigar futuros impactos ambientais causados pela aplicação excessiva de fertilizantes, especialmente de nitrogênio (Costa et al., 2016), além de economia de recursos financeiros, recomenda-se que esta quantidade aportada pela Pi seja reduzida da aplicação via fertilizante mineral.

O enxofre não foi adicionado na fertilização mineral, nas fases de plantio e cobertura, no entanto as entradas pela $\mathrm{Pi}$ fornecem o suprimento necessário, uma vez que não há comprometimento do crescimento das árvores e expressão de sintomas de deficiência de $S$ no povoamento de Eucalyptus saligna. Este estudo evidencia a importante contribuição da precipitação pluviométrica na ciclagem de nutrientes e nutrição florestal, seja para reduzir a fertilização mineral com nitrogênio ou suprir as necessidades da planta por enxofre.

Em função do menor volume de precipitação anual que incidiu sobre a plantação de Eucalyptus saligna, o aporte de N e S é superior ao observado em Dick et al. (2018). Na região oeste do bioma Pampa, durante o ano de 2013, os referidos autores registraram o volume de precipitação anual de $1.636,9 \mathrm{~mm}$, que aportou em um povoamento de Eucalyptus dunnii 0,50, 1,25 e 0,0007 kg ha-1 ano-1 de $\mathrm{N}$ (nitrato), pela P, Pi e Et, respectivamente. Também houve entrada de menores quantidades de enxofre, com 2,01, 1,99 e 0,045 $\mathrm{kg} \mathrm{ha}^{-1}$ ano $^{-1}$ pela P, Pi e Et, respectivamente. 
Em função da ampla variação nas concentrações de nutrientes, considerar o volume de precipitação pluviométrica incidente sobre os povoamentos florestais, visando às estimativas de aporte, é imprescindível para o entendimento da ciclagem de nutrientes. Para tanto, é necessário e recomendável que se realize o monitoramento da ciclagem geoquímica por longo prazo, considerando as diferentes formas de distribuição, concentrações de nutrientes e volumes de precipitação pluviométrica nos mais variados ecossistemas florestais.

\section{CONCLUSÕES}

No povoamento de Eucalyptus saligna a concentração de nitrato é maior na precipitação interna, seguido de precipitação incidente e escoamento pelo tronco, ao passo que a forma de distribuição não influenciou a concentração de sulfato.

Há relevante contribuição da precipitação interna para a ciclagem geoquímica de nitrogênio e da precipitação incidente para o aporte de enxofre em povoamento de Eucalyptus saligna. Estratégias de manejo da fertilização mineral, para a silvicultura na região central do Rio Grande do Sul, bioma Pampa, podem ser adotadas com base nos resultados do presente estudo. As recomendações futuras de aplicação serão otimizadas por meio da redução de até $36,8 \%$ de N, reduzindo a aplicação excessiva de nutrientes e custos nas operações florestais, minimizando futuros impactos ambientais.

\section{AGRADECIMENTOS}

Agradecimento à Empresa CMPC pelo apoio logístico e disponibilidade da área de estudo.

\section{REFERÊNCIAS}

Almeida, A. C., Soares, J. V., Landsberg, J. J., \& Rezende, G. D. (2007). Growth and water balance of Eucalyptus grandis hybrid plantations in Brazil during a rotation for pulp production. Forest Ecology and Management, 251(1-2), 10-21.

Alvares, C. A., Stape, J. L., Sentelhas, P. C., Gonçalves, J. L. M., \& Sparovek, G. (2013). Köppen's climate classification map for Brazil. Meteorologische Zeitschrift, 22(6), 711-728.

American Public Health Association - APHA. (1998). Standard methods for the examination of water and wastewater (19th ed.). Washington: American Public Health Association, American Water Works Association, Water Pollution Control Federation.

Ashagrie, Y., \& Zech, W. (2010). Dynamics of dissolved nutrients in forest floor leachates: comparison of a natural forest ecosystem with monoculture of tree species plantations in south-east Ethiopia. Ecohydrology \& Hydrobiology, 10(2-4), 183-190.

Begon, M., Townsend, C. R., \& Harper, J. L. (2007). Ecologia: de indivíduos a ecossistemas (4. ed). Porto Alegre: Artmed.

Carnol, M., \& Bazgir, M. (2013). Nutrient return to the forest floor through litter and throughfall under 7 forest species after conversion from Norway spruce. Forest Ecology and Management, 309, 66-75.

Chang, M. (2006). Forest hydrology: an introduction to water and forests (2nd ed.). USA: Taylor \& Francis Group.

Costa, D. D., Kempka, A. P., \& Skoronski, E. (2016). A contaminação de mananciais de abastecimento pelo nitrato: o panorama do problema no Brasil, suas consequências e as soluções potenciais. Revista Eletrônica do PRODEMA, 10(2), 49-61.

Dick, G., Schumacher, M. V., Momolli, D. R., \& Viera, M. (2018). Nutrient input via incident rainfall in a Eucalyptus dunnii stand in the Pampa biome. Floresta e Ambiente, 25(3), e20160559.

Diniz, A. R., Pereira, M. G., Balieiro, F. C., Machado, D. L., \& Menezes, C. E. G. (2013). Precipitação e aporte de nutrientes em diferentes estádios sucessionais de Floresta Atlântica, Pinheiral - RJ. Ciência Florestal, 23(3), 389-399.

Giglio, J. N., \& Kobiyama, M. (2013). Interceptação da chuva: uma revisão com ênfase no monitoramento em florestas brasileiras. Revista Brasileira de Recursos Hídricos, 18(2), 297-317.

Instituto Nacional de Meteorologia - INMET. (2016). Recuperado em 12 de janeiro de 2016, de http://www.inmet.gov.br/portal/ 
Laclau, J.-P., Ranger, J., Bouillet, J.-P., Dieu Nzila, J., \& Deleporte, P. (2003). Nutrient cycling in a clonal stand of Eucalyptus and an adjacent savanna ecosystem in Congo 3: chemical composition of rainfall, throughfall and stemflow solutions. Forest Ecology and Management, 176(1-3), 105-119. http://dx.doi.org/10.1016/S0378-1127(02)00280-3.

Laclau, J.-P., Ranger, J., Gonçalves, J. L. M., Maquère, V., Krusche, A. V., M'Bou, A. T., Nouvellon, Y., SaintAndré, L., Bouillet, J.-P., Piccolo, M. C., \& Deleporte, P. (2010). Productivity in Tropical Plantations Biogeochemical cycles of nutrients in tropical Eucalyptus plantations: main features shown by intensive monitoring in Congo and Brazil. Forest Ecology and Management, 259(9), 1771-1785. http://dx.doi.org/10.1016/j.foreco.2009.06.010.

Lima, W. P. L. (1976). Interceptação da chuva em povoamentos de eucalipto e de pinheiro. Scientia Forestalis IPEF, (13), 75-90.

Melo, A. B. C. (2011). Previsão de chuvas acima da normal para o norte do Brasil (Boletim de Informações Climáticas do CPTEC/INPE, Vol. 18, No. 11). MCT/INPE/CPTEC.

Poggiani, F., \& Schumacher, M. V. (2005). Ciclagem de nutrientes em florestas nativas. In J. L. M. Gonçalves \& V. Benedetti (Eds.), Nutrição e fertilização florestal. Piracicaba: IPEF.

Salvador, S. M., Consensa, C. B., \& Araújo, E. F. (2014). Produção de serapilheira e devolução de macronutrientes em um povoamento de Eucalyptus saligna (F. Muell). Ecologia e Nutrição Florestal, 2(2), 52-62.

Salvador, S. M., Schumacher, M. V., Viera, M., Stahl, J., \& Consensa, C. B. (2016). Biomassa e estoque de nutrientes em plantios clonais de Eucalyptus saligna Smith. em diferentes idades. Scientia Forestalis, 44(110), 311-321.

Sari, V., Paiva, E. M. C., \& Paiva, J. B. D. (2016). Interceptação da chuva em diferentes formações florestais na região sul do Brasil. Revista Brasileira de Recursos Hídricos, 21(1), 65-79.

Schumacher, M. V., \& Viera, M. (2015). Ciclagem de nutrientes em plantações de eucalipto. In M. V. Schumacher \& M. Viera (Eds.), Silvicultura do Eucalipto no Brasil. Santa Maria: Editora UFSM.

Shen, W., Ren, H., Darrel Jenerette, G., Hui, D., \& Ren, H. (2013). Atmospheric deposition and canopy exchange of anions and cations in two plantation forests under acid rain influence. Atmospheric Environment, 64, 242-250.

Wu, D., Wang, S., Xia, J., Meng, X., Shang, K., Xie, Y., \& Wang, R. (2013). The influence of dust events on precipitation acidity in China. Atmospheric Environment, 79, 138-146.

Contribuição dos autores: GD: conceituação, curadoria de dados investigação, metodologia, visualização, escrita - primeira redação, escrita - revisão e edição. MVS: conceituação, curadoria de dados, obtenção de financiamento, metodologia, administração do projeto, supervisão, visualização; EFA: conceituação, curadoria de dados, obtenção de financiamento, metodologia, supervisão, visualização. 